e-Neuroforum 2011 · 2:61-67

DOI 10.1007/s13295-011-0019-0

(c) Springer-Verlag 2011
T. Kuner ${ }^{1} \cdot$ A. Schaefer ${ }^{2}$

${ }^{1}$ Anatomie und Zellbiologie, Universität Heidelberg, Heidelberg

2 Max-Planck-Institut für medizinische Forschung, Heidelberg

\title{
Molecules, cells and networks involved in processing olfactory stimuli in the mouse olfactory bulb
}

\section{From stimulus to behaviour- the olfactory system's ability to discriminate}

For many animals, in particular nocturnal creatures, olfaction is a sensory modality of fundamental importance. The ability to identify and distinguish between odours is in many instances essential to survival. Olfaction serves not only in the detection of potentially poisonous or particularly tasty food, but is also used to communicate or navigate. Common to these two functions is the fact that they both need to compare and recognize odours. Which mechanisms confer these abilities on the olfactory system? Which anatomical, cellular and molecular conditions permit the discrimination of odours?

\section{The olfactory stimulus and its representation in the mouse olfactory bulb}

From a chemical perspective, most odours comprise a mixture of several up to hundreds of different odorants. Each of these monomolecular odorants, e.g. amyl acetate (AA) or ethyl butyrate (EB), binds with varying affinity to many of the 1200 olfactory receptors, of which each olfactory receptor neuron expresses only one receptor type [1]. Thus each odorant activates an individual combination of olfactory receptor neurons, each with varying strength. The spatially and temporally organized pattern of electrical activity produced in this way is the first representation of an odorant in the olfactory system. By means of $\mathrm{Ca}^{2+}$ imaging in anesthetised mice, these odorant-evoked affer- ent activity patterns can be imaged with good spatio-temporal resolution. Voltagesensitive dye imaging in the olfactory bulb [2] demonstrates that the pure substances AA and EB are represented by different patterns, whereby only the spatial aspect is shown here (- Fig. 1). The different patterns suggest that the mouse can easily distinguish between amyl acetate and ethyl butyrate.

This proposal can be investigated in behavioural experiments (• Fig. 2), whereby mice learn to discriminate between odorants in an operant conditioning test [3]. In order to effectively make the association between the perception of an odorant and obtaining a reward, mice need to be exposed to an odorant between 500 and 1000 times. The mouse can then identify the trained odorant with a reliability of $95 \%$ or more (• Fig. 1 ). This corresponds to our intuitive expectations in the case of two pure substances; however, can the mouse also identify other similar stimuli just as effectively? To artificially generate similar stimuli, binary mixtures of the two substances can be produced: a mixture of $60 \%$ AA and $40 \% \mathrm{~EB}$ is compared with a mixture of $40 \%$ AA and $60 \%$ EB. By converging the percentages (e.g. $52 \% / 48 \%$ versus $48 \% / 52 \%$, in each case the whole concentration of odorant being $1 \%$ in mineral oil), similar stimuli can be produced. In contrast to the pure-substance patterns, binary mixtures demonstrate highly similar patterns with only minimal differences as expected (• Fig. 1). Surprisingly, these stimuli can also be discriminated with an almost perfect reliability of $95 \%$ and more (• Fig. 2D), whereby acquiring this ability takes somewhat longer than does simple-odour discrimination (• Fig. 1D). Which other parameters of behavioural output could be altered? To answer this question, we measured an established variable of sensory physiology, i.e. the time needed to discriminate between two stimuli [3]. This reaction time, referred to here as discrimination time, provides direct information about the neuronal processing procedures on which the ability to discriminate is based (- Fig. 2). Measuring the discrimination times for pure substances and binary mixtures shows that simple odours can be discriminated in as little as $240 \mathrm{~ms}$, while approximately $100 \mathrm{~ms}$ more time is needed for the mixtures ( $\bullet$ Fig. 1). Longer discrimination times for binary mixtures were seen for all odorant pairs tested to date. These observations suggest that, in order to discriminate between similar odours, neuronal processing operations take place in the olfactory system which require time to distinguish differences in the afferent activity pattern, thus enabling successful olfactory discrimination behaviour. Which neuronal mechanisms underlie these operations and require up to $100 \mathrm{~ms}$ more processing time?

\section{Anatomy of the olfactory system}

In order to answer this question, we need to consider the anatomy of the mouse olfactory system (- Fig. 3). The bulb is bordered externally by the olfactory nerve layer and comprises the glomerular layer, the external plexiform layer, the mitral cell layer, the internal plexiform layer and the central granule cell layer. The glomerular layer comprises a multitude of spher- 


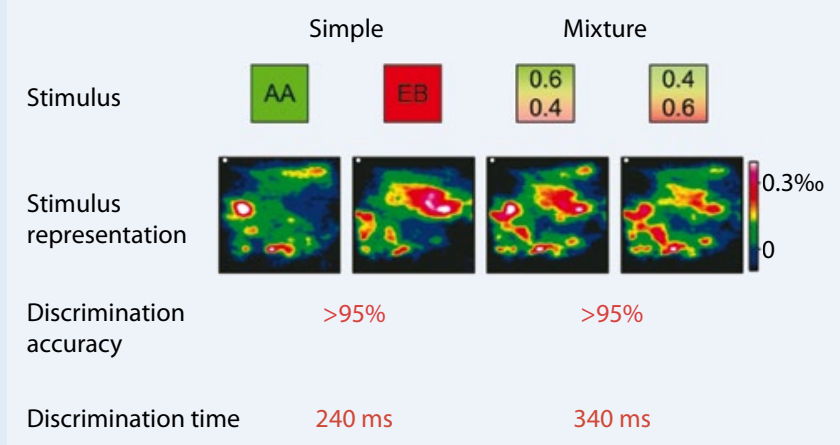

Fig. $1 \Delta$ Stimulus, stimulus representation and stimulus discrimination. The monomolecular odorants amyl acetate $(A A)$ and ethyl butyrate $(E B)$ and their"binary" mixtures (60\% AA and $40 \%$ EB with a total concentration of $1 \%$ odorant in mineral oil). The similarity between stimuli is visually represented in the form of colour scaling. In vivo imaging with voltage-sensitive dye imaging. During the experiment, these are applied directly to the surface of the brain via cranioectomy and become embedded in the nerve cell membrane. A change in membrane tension then results in altered fluorescence, which can be measured with good spatio-temporal resolution directly from the dorsal surface of the bulb. The image shows one time point only a few hundred milliseconds following odorant application. Colour scaling ranges from 0 to 0.4 per mille in intensity modulation following stimulus application relative to the background (warm colours indicate regions of greater activation). The image shows a large portion of the surface area of the dorsal olfactory bulb, while the left margin corresponds to the anterior and the upper margin to the lateral borders. The white disk in the left upper corner roughly represents the size of a glomerulus with a diameter of $80 \mu \mathrm{m}$. The reliability with which the mouse makes a correct decision is shown in percent, while the time needed for this decision is given in milliseconds. (Modified after [3], Elsevier @ 2004)

ical glomeruli, which are a specialization of the neuropil and comprise interneuron and principal cell (mitral and tufted) dendrites, as well as olfactory receptor neuron nerve endings of the olfactory epithelium. The latter project into the glomeruli where they form synapses with interneuron and principal cell dendrites. Olfactory receptor neurons that express identical olfactory receptors project into one or fewer (in general <4) glomeruli, even if they are distributed over a large surface area of the olfactory epithelium (• Fig. 3A). Thus a glomerulus can be considered an afferent structure that unites information from one receptor type, thus representing a form of "input channel". The arrangement of the glomeruli in the superficial layer of the olfactory bulb produces a spatial map of the input channels. Every odour generates an individual spatial activity pattern (• Fig. 1).

Each glomerulus has approximately ten mitral cells containing one apical dendrite and several lateral dendrites. Their apical dendrites located within the glom- erulus receive olfactory receptor neuron axons, but are also contacted by periglomerular cells and other juxtaglomerular cells, in particular the so-called short-axon cells. This produces an initial local processing level; activity in the neighbouring glomeruli can be compared by means of the short-axon cells (these mechanisms are not discussed in greater detail here). Processing between various glomeruli takes place primarily via granule cells in deep layers [4]. Granule cells are axonless neurons and are specialized in dendrodendritic communication [5]: granule cell dendrites form synapses with the lateral dendrites of mitral cells. The lateral dendrites can be significant in length, traversing a large proportion of the olfactory bulb. Mitral cell axons travel towards higher brain regions, such as the piriform cortex or the hippocampus, and transport the processed output signals from the olfactory bulb (- Fig. 3)

Dendrodendritic synapses, also referred to as reciprocal synapses, comprise two opposingly arranged synaptic con- tacts of varying polarity [6] (• Fig. 3B): an asymmetrical (Gray type I) glutamatergic contact in the granule cell direction and a symmetric (Gray type II) GABAergic contact in the mitral cell direction. By virtue of this arrangement, presynaptic excitatory and postsynaptic inhibitory structural elements lie directly adjacent to one another in lateral dendrites, and vice versa in granule cell dendrites.

Action potentials released in $\mathrm{mi}-$ tral cells extend to lateral dendrites [7, 8], where they cause the release of the excitatory neurotransmitter glutamate (- Fig. 3B, 1). This binds to postsynaptic AMPA and NMDA receptors on granule cell dendrites [9], resulting in depolarisation and $\mathrm{Ca}^{2+}$ influx, which in turn cause the release of the inhibitory neurotransmitter GABA back to the stimulating mitral cell (• Fig. 3B, 2, recurrent inhibition), as well as to the dendrites of other mitral cells (- Fig. 2B, 3, lateral inhibition).

\section{Inhibitory networks}

This type of anatomical consideration suggests that inhibitory processing in the olfactory bulb is of particular importance. Indeed, physiological measurements show that the olfactory response of mitral cells is not only excitatory in nature, but can also be inhibitory $[8,10,11,12,13]$. Thus, from a series of similar odours (e.g. aldehyde of varying chain length; - Fig. 4A [13]), those odours inducing the maximal response are often "flanked" by odours which evoke inhibitory responses $\left(\mathrm{C}_{4}\right.$, C8). This suggests a type of lateral inhibition in olfaction similar to retinal processing $[11,14,15]$. Indeed, intracellular recordings of mitral cells partially confirm this view: Luo and Katz recorded targeted mitral cell signals and correlated the excitatory or inhibitory mitral cell response to various odours with the "maps" which could be measured using imaging methods (- Fig. 4B) $[2,12,16]$. In this context, they initially noticed that, as expected, mitral cells are strongly depolarized by those odours which had also shown local activity in imaging experiments. In good agreement with the anatomical conditions, however, the authors could also measure inhibitory receptive fields in the 


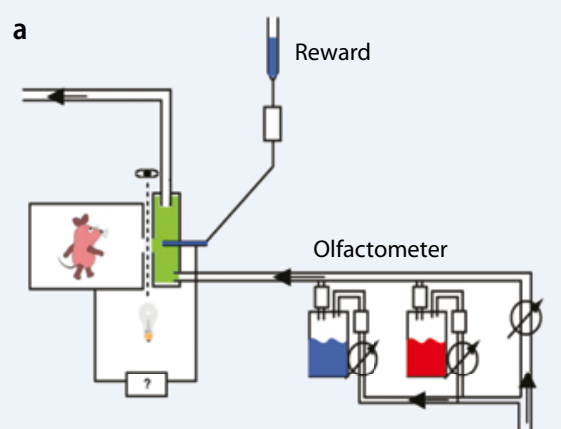

b

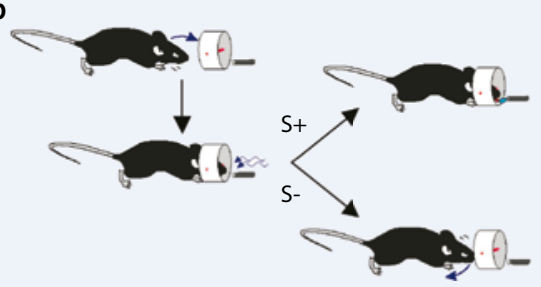

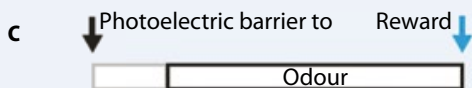

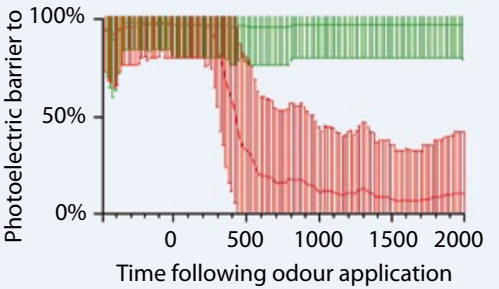

d

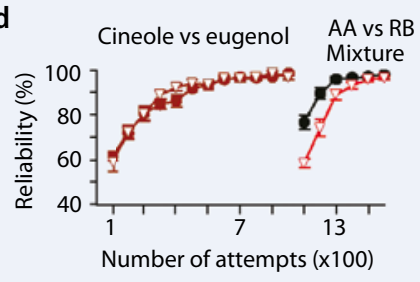

Fig. $2 \Delta$ Olfactory discrimination behaviour. A number of behavioural tests are available to investigate the capacity of the mouse olfactory system. Its representation here is restricted to the go/no-go paradigm of an operant conditioning test [31]. A Behavioural test design: Using an olfactometer, electrically controlled solenoid valves can produce odour pulses of defined duration and concentration ( $1 \%$ odorant in mineral oil, blue and red). An air stream with a defined flow velocity takes up the saturated odorant atmosphere via the mineral oil, mixes with the carrier air stream and enters the olfactory cylinder (green), which contains a metal tube to deliver water as a reward (blue). The olfactory cylinder is connected to the test chamber by an opening controlled by a photoelectric barrier (light bulb and eye symbols). When the mouse is rewarded for licking the metal tube, this can be measured via an air stream between the metal floor of the chamber and the tube. These processes, as well as measured data acquisition (photoelectric barrier, licking), are controlled automatically via a PC with appropriate software. B Behavioural experiment procedure: The mice are kept under water restriction to increase their perception of water as a reward during behavioural tests. At first, the mice familiarize themselves with the apparatus and, by actively exploring, quickly find the site at which water is provided as a reward. The animals then learn the association between reward and odorant. This is followed by the actual experimental sessions (100-200 trials/day). Breaching the photoelectric barrier (B, red) causes an odorant to be applied following a short delay (curvedblue arrow) until water (blue droplets) is released via a valve (see $\mathbf{C}$ for the time course). In the case of the rewarded odorant $(O+)$, the mouse waits for the reward with its head in the olfactory cylinder (photoelectric barrier unbreached); in the case of an unrewarded odorant $\left(O_{-}\right)$, the mouse stereotypically retracts its head from the olfactory cylinder (the photoelectric barrier is closed, bottom right in B). The next trial can be started following an interval of $5 \mathrm{~s}$. Odorants are applied in a random sequence. C Measurement parameters and their evaluation: By monitoring the photoelectric barrier, it is possible to measure with high temporal resolution whether or not the mouse's head is in the olfactory cylinder. In the case of a rewarded odorant, the mouse usually leaves its head in the olfactory cylinder (green $\mathrm{O}+$ curve, mean \pm standard deviation. The distribution of values results from rare incorrect decisions made by the mice). In the case of unrewarded odorants, the animal usually retracts its head rapidly (red $\mathrm{O}$ - curve, mean \pm standard deviation). It can be determined from approximately 100 trials at which point in time the $\mathrm{O}+$ and $\mathrm{O}-$ curves differ significantly. This yields the olfactory discrimination time. The time between individual trials provides information on the mouse's motivational status and is important for the interpretation of experiments. Measuring licking behaviour shows on the one hand whether the reward has been accepted and whether acquisition has taken place, while on the other it provides additional information on the mouse's motivational status. The mouse should only lick on application of a rewarded odorant, otherwise responses will be counted as incorrect. Thus the number of correct decisions (reliability) can be determined and represented in a learning curve (D). When initially learning to discriminate between cineole and eugenol, the mice need $800-1200$ trials to achieve a correct-response rate of $95 \%$. Thereafter, amyl acetate $(A A)$ and ethyl butyrate $(E B)$ can be highly reliably distinguished after only 300 trials. Acquiring binary mixtures requires somewhat more time. Odour acquisition can be tested by means of intermittent testing with odorant pairs which have already been acquired. (Modified from [3], Elsevier @ 2004)

region of the recorded cell. Both investigations demonstrate the particular role of inhibition in the activity and spatial activity pattern.
In order to investigate to what extent olfactory bulb interconnections influence the temporal dynamics of the olfactory response, Albeanu et al. recently investigat-
e-Neuroforum $2011 \cdot 2: 61-67$

DOI 10.1007/s13295-011-0019-0

○) Springer-Verlag 2011

T. Kuner $\cdot$ A. Schaefer

Molecules, cells and networks involved in processing olfactory stimuli in the mouse olfactory bulb

\section{Abstract}

How sensory stimuli are processed by neural networks is a key question of neuroscience. Olfactory conditioning experiments in mice demonstrated that odour processing is fast and stimulus-dependent. Selective genetic perturbation of the inhibitory circuitry in the first relay station of olfactory processing, the olfactory bulb, altered such discrimination times, with increased inhibition accelerating and decreased inhibition slowing down odour discrimination. This illustrates that inhibition fulfils a key role in sensory processing.

\section{Keywords}

Olfaction · Olfactory bulb · Physiology · Odour discrimination · Inhibition 


\section{Review article}

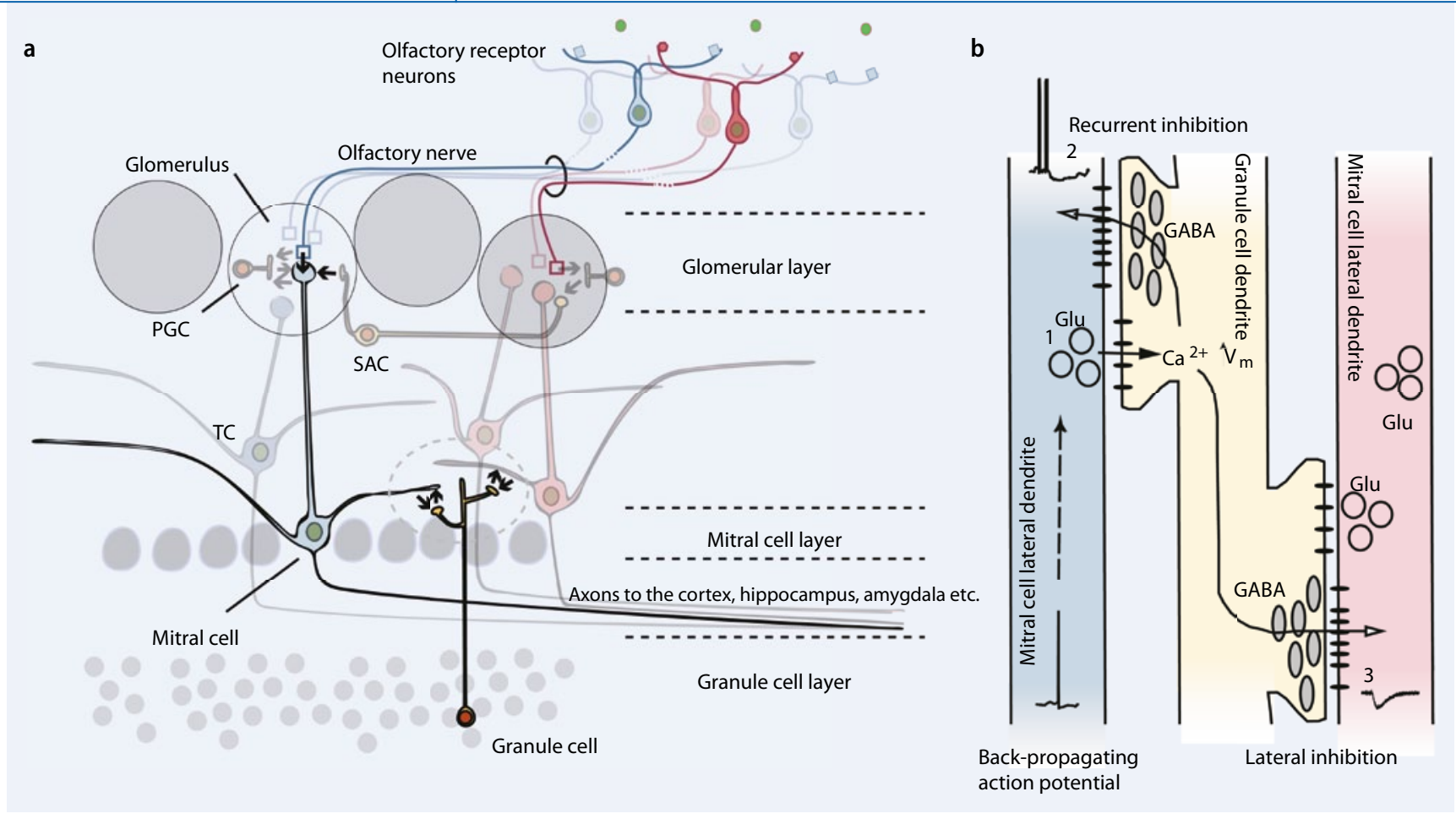

Fig. $3 \Delta$ Anatomical structure of the olfactory bulb. A Layers and cell types of the bulb, olfactory nerve and olfactory epithelium (top right). Glomeruli are round neuropil structures $(80 \mu \mathrm{m}$ in diameter) made up of interneurons ( $P G C$ periglomerular cells, SAC short-axon cells), the apical dendrites of the mitral and tufted cells (TC) as well as olfactory receptor neuron endings. SACs connect neighbouring glomeruli and sometimes have very long axons - contrary to their historical name. Olfactory receptor neurons marked in the same colour have one receptor type and project to the same glomerulus. B Functional architecture of the dendrodendritic synapse (grey dotted region in $\mathbf{A}$ ). Lateral dendrites of the mitral cells shown in $\mathbf{A}$ (blue and red) and granule cells (yellow). Oval synaptic vesicles contain GABA, while round synaptic vesicles contain glutamate as a neurotransmitter. Arrows indicate the signal flow: 1 release of the excitatory neurotransmitter glutamate, 2 recurrent inhibition, 3 lateral inhibition

ed specifically those mitral cells which receive input signals from the same glomerulus [17]. These"sister" mitral cells actually show very similar olfactory profiles to those expected on the basis of the genetic identity of the olfactory fibres converging in the glomerulus $[18,19]$. However, on closer analysis, the authors observed significant differences in the temporal dynamics of the olfactory response, in particular in the phase of olfactory response relating to inhalation cycles $[20,21$, 22, 23] (• Fig. 4C, [17]). This can be explained in turn by the varying lateral interconnection.

These and other physiological findings, together with detailed knowledge of anatomy [24], support the hypothesis that inhibition plays a particular role in olfactory processing and possibly that the additional time needed to process similar odorants is at least partially attributable to processes in this inhibitory network.

In order to pursue this hypothesis, it would be necessary to undertake a target- ed modification in the granule cell network and follow the results of such a modification analytically through the various descriptive levels-cell, network, behaviour. Perhaps the greatest challenge in this context would be to precisely control the intervention spatially and temporally. Its restriction to a defined anatomical space is necessary to modify solely the granule cell network in question, without disrupting any of those areas of the brain relevant to performing movements or necessary for general decision-making processes. Moreover, the timing of the intervention is crucial, since the mouse goes through many well-coordinated steps of maturation on its way from an embryo to an adult organism which, if disrupted by an intervention, could also cause unwanted and adverse side effects. However, genetic modifications, e.g. the targeted removal of individual genes, generally manipulate target genes in all body cells, often as early on as during prenatal development. Modern genetic techniques have made it possible to flank an individual gene with special interfaces, so-called loxP sites [25]. When Cre-recombinase, a bacteriophage enzyme, is expressed in a cell it functions likes molecular scissors which irreversibly cut out the gene on the loxP site. By spatially controlling the expression of Cre-recombinase and restricting it, for example, to groups of neurons, genetic modifications can in principle be restricted to precisely these groups of neurons [26]. The time point at which Cre-recombinase is expressed in turn determines at what stage of mouse development the intervention takes place. To apply this to the olfactory bulb granule cells, a promotor needs to be found which is expressed exclusively in granule cells and then only in adulthood. Although it is theoretically possible to find this type of specific promotor, it is generally highly unlikely. Alternatively, there is the option to introduce viruses which, as gene carriers, carry information on the production of Cre-recombinase in a targeted manner by means of stereotac- 

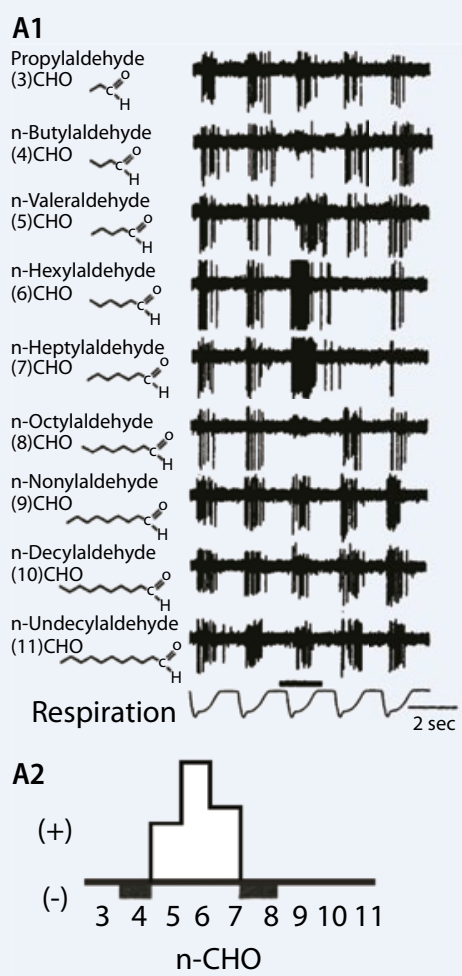

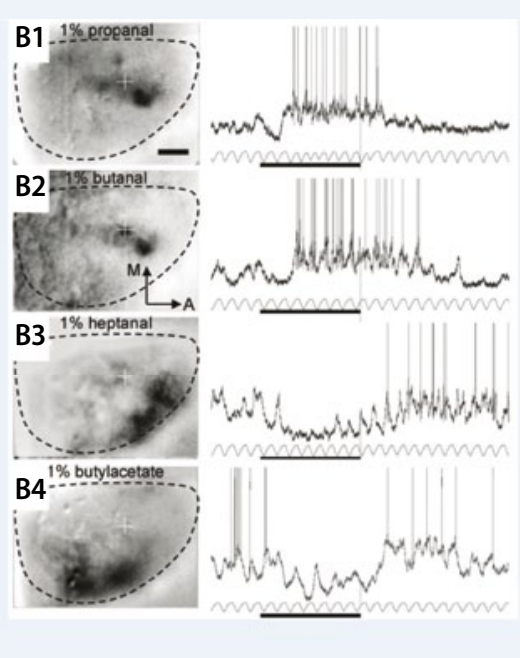

B5

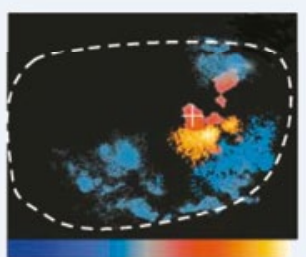

C1

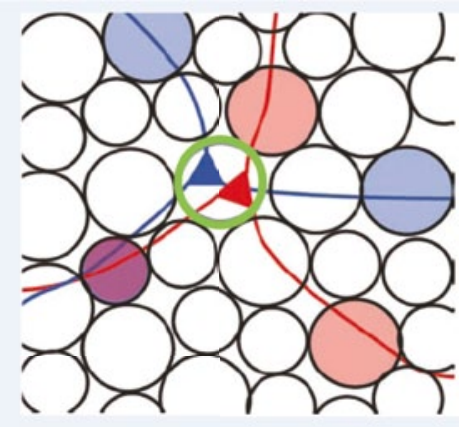

C2

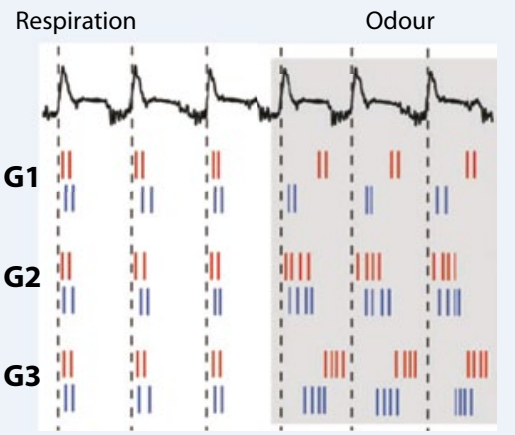

Fig. $4 \Delta$ A Extracellular measurement of action potential propagation of mitral cells in vivo. The cell shown reacts most strongly to stimulation (bars) with aldehydes with between five and seven carbon atoms; "neighbouring" odorants, i.e. aldehyde with a chain length of 4-8 carbons, inhibit spontaneous cell activity. A2 The generation rate as a function of the olfactory stimulus (modified from [13]). B1-B4 Spatial activity pattern for four different odorants (imaging using intrinsic signals from the dorsal side of the olfactory bulb in an anesthetized mouse). Right Electrical activity in a mitral cell (identical cells in 1-4) at the position marked with a white cross (+). B5 A summary of B1-B4 with regions which preferentially evoked inhibitory (red, yellow, see also e.g. B1, B2) or excitatory responses in the mitral cells measured (blue, e.g. B3, B4) (modified from [12], Elsevier $\odot 2001$ ). C1 Two"sister" mitral cells whose primary dendrites receive input signals from the same glomerulus (green circle). Their olfactory response varies by virtue of the fact that both mitral cells are affected by different glomeruli (mediated via other mitral cells and granule cells, see - Fig. 2A) via their lateral dendrites. C2 This leads to different olfactory responses in two"sister" mitral cells. Differences are particularly visible in the action potential generation phase relative to the respiration cycle (modified from [17], Macmillian Publishers Ltd. on behalf of Cancer Research UK @ 2010)

tic injections in the centre of the olfactory bulb of adolescent mice ( $\bullet$ Fig. 5,6$)$. In this way, any target genes can be specifically eliminated, assuming there is a mouse line in which the target gene is flanked by loxP interfaces. Although producing a"floxed" mouse line of this kind is complex and costly, many laboratories choose to do so due to the significant experimental advantages it offers.

Special target genes that are important in the synaptic transmission of mitral cells to granule cells are suited to modifying inhibitory interconnections. In this context, we were able to show that-as known from other areas of the brain-removal of the GluA2 subunit results in an increased influx of calcium ions into granule cells (• Fig. 6, B1-B3) [27]. This in turn induces a marked increase in the inhibition of mitral cells, as one would expect on the basis of the tortuous architecture of dendrodendritic synapses (- Fig. 3, 6 , B4) $[7,9,27,28]$. By thus investigating mice with targeted genetic modifications in terms of their ability to discriminate odours, it becomes evident that the acquisition and storing of olfactory information remains unchanged between control groups, and that general behavioural tests show no differences due to the highly regional modifications. Measurements of processing speed, however, demonstrated marked changes (• Fig. 6, B5) [27]: although simple odorant discrimination took as long as in controls, the genetically modified animals with increased inhibition required significantly less time to discriminate highly similar odorant mixtures; they were faster than their control conspecifics in the same experiment! A similar modification to the GluN1 sub- unit, which lead to reduced calcium influx and hence reduced inhibition (• Fig. 6, $\mathrm{C}_{1}-\mathrm{C}_{4}$, - Fig. 3B), showed precisely the opposite effect: in the case of unchanged ability to acquire and memorize, the discrimination of similar odours required significantly longer (• Fig. 6, C5) [27].

In conjunction with other physiological and behavioural experiments in genetically modified animals $[29,30]$, these investigations show that the inhibitory network of the olfactory bulb is indeed of considerable significance in olfactory processing. Most memory tasks-at least simple olfactory discrimination paradigmscan be attributed in turn to the olfactory cortex and, in particular, to the plasticity of the piriform cortex. 


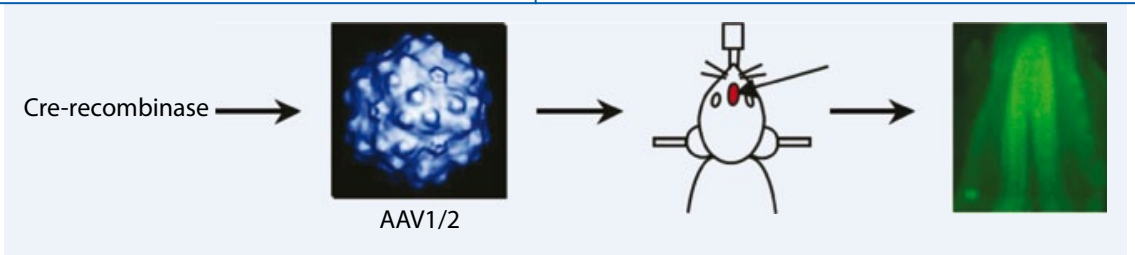

Fig. $5 \Delta$ Viral gene transfer and stereotaxis. Viruses have become powerful tools for molecular interventions in the nervous system over the last 10 years, whereby adeno-associated viruses (AAV), adenoviruses and lentiviruses are preferentially used. Furthermore, herpes viruses, alpha viruses and pseudo-rabies viruses are also used. Each of these virus systems possesses a characteristic profile of properties, which is decisive when choosing the appropriate system. A feature common to all viruses is that they can be subjected to an almost limitless arsenal of molecular genetic interventions to manipulate neurons in a targeted manner. These include, among others, simple overexpression of proteins, the use of RNAi, toxins, Cre-recombinase, conditional expression cassettes and channel rhodopsins. These methods can be combined in genetically manipulated mice, as a result of which the number of possibilities multiplies (e.g. the combination of conditional mouse lines with viral expression of Cre-recombinase, see main text). Viruses are also excellently suited to performing initial experiments in reduced model systems, such as cell cultures, before scaling them up to the level of the intact organism. A pathway for targeted virus administration is required in order to use viruses in the central nervous system of a living organism. Stereotactic injection of viral suspensions in defined areas of the brain is particularly suited to this end. Using this method, and depending on the virus characteristics, very small brain areas (e.g. thalamic nuclei) or entire regions (e.g. granule cell layer of the olfactory bulb) can be infected with great efficiency (up to $95 \%$ of expressing cells). Furthermore, conditions can be created under which only scant neurons are infected. The procedure for a viral in vivo gene transfer experiment includes cloning the desired construct, producing and purifying infectious particles, as well as stereotactic intervention and injection of the virus suspension in the target area and at the time of expression (depending on the system, $12 \mathrm{~h}-12$ days). This cycle can generally be achieved within 4 weeks, providing a highly flexible and easily adaptable method

\section{Corresponding address \\ T. Kuner}

Anatomie und Zellbiologie,

Universität Heidelberg

Im Neuenheimer Feld 307, 69120 Heidelberg

Germany

kuner@uni-heidelberg.de

T. Kuner 1988-1998: Medical studies, Ruprecht-Karls University, Heidelberg, on a scholarship from the German National Academic Foundation.1992-1997: Doctoral thesis under Prof. Dr. Peter H. Seeburg, Center for Molecular Biology at the University of Heidelberg (ZMBH).1998-2000: Post-doctoral student under Prof. George Augustine, Duke University Medical Center, Durham, NC and Marine Biological Laboratory, Woods Hole, MA, USA; Feodor-Lynen scholarship from the Alexander von Humboldt Foundation, scholarship from the "Human Frontiers in Science Program", Grass Fellowship in Neurosciences.2000-2006: Principal Investigator, Department of Cell Physiology, Prof. Dr. Bert Sakmann, Max-Planck Institute for Medical Research, Heidelberg; Claussen-Simon Foundation scholarship.2002-2005: Principal Investigator in the interdisciplinary research group "WIN-Olfactory Dynamics Group" and programme of the Heidelberg Academy of Sciences.2003: Awarded Professorship in Physiology, Heidelberg University. Since 2006: Professor at the Institute for Anatomy and Cell Biology, Heidelberg University.

\section{A. Schaefer \\ Max-Planck-Institut für medizinische Forschung Heidelberg \\ Germany}

A. Schaefer 1995-2000: Studied Physics (subsidiary subject: Cell Biology) at the Ruprecht-Karls University, Heidelberg, on a scholarship from the German National Academic Foundation.2000-2001: Research associate, Department of Experimental Psychology, Oxford, UK.2001-2004: PhD in Biology, MPI for Medical Research, University of Heidelberg, under Bert Sakmann on a scholarship from the Boehringer Ingelheim Fonds and the "WIN Kolleg" programme of the Heidelberg Academy of Sciences.2004-2007: Postdoctoral student under Troy Margrie, Department of Physiology, University College London, UK, supported by the Leopoldina Academy of Sciences and EMB0.2007-2008: David Phillips Fellow and Principal Investigator, Department of Physiology, UCL, UKSince 2008: Max Planck Research Group Leader, MPI for Medical Research, Heidelberg2009: Appointment to the Research Professorship for Neurosciences, Ruprecht-Karls University Heidelberg.

\section{References}

1. Mombaerts P (2004) Genes and ligands for odorant, vomeronasal and taste receptors. Nat Rev Neurosci 5:263-278

2. Spors H, Grinvald A (2002) Spatio-temporal dynamics of odor representations in the mammalian olfactory bulb. Neuron 34:301-315

3. Abraham NM, Spors H, Carleton A et al. (2004) Maintaining accuracy at the expense of speed: stimulus similarity defines odor discrimination time in mice. Neuron 44:865-876
4. Aungst JL, Heyward PM, Puche AC et al. (2003) Centre-surround inhibition among olfactory bulb glomeruli. Nature 426:623-629

5. Shepherd GM, Chen WR, Willhite D, Migliore M, Greer CA (2007) The olfactory granule cell: from classical enigma to central role in olfactory processing. Brain Res Rev 55:373-382

6. Rall W, Shepherd GM, Reese TS, Brightman MW (1966) Dendrodendritic synaptic pathway for inhibition in the olfactory bulb. Exp Neurol 14:44-56

7. Chen WR, Xiong W, Shepherd GM (2000) Analysis of relations between NMDA receptors and GABA release at olfactory bulb reciprocal synapses. Neuron 25:625-633

8. Margrie TW, Sakmann B, Urban NN (2001) Action potential propagation in mitral cell lateral dendrites is decremental and controls recurrent and lateral inhibition in the mammalian olfactory bulb. Proc Natl Acad Sci U S A 98:319-324

9. Isaacson JS, Strowbridge BW (1998) Olfactory reciprocal synapses: dendritic signaling in the CNS. Neuron 20:749-761

10. Kauer JS (1974) Response patterns of amphibian olfactory bulb neurones to odour stimulation. J Physiol 243:695-715

11. Laurent $G$ (1999) A systems perspective on early olfactory coding. Science 286:723-728

12. Luo M, Katz LC (2001) Response correlation maps of neurons in the mammalian olfactory bulb. Neuron 32:1165-1179

13. Yokoi M, Mori K, Nakanishi S (1995) Refinement of odor molecule tuning by dendrodendritic synaptic inhibition in the olfactory bulb. Proc Natl Acad Sci U SA 92:3371-3375

14. DeVries SH, Baylor DA (1993) Synaptic circuitry of the retina and olfactory bulb. Cell 72 [Suppl]:139-149

15. Urban NN (2002) Lateral inhibition in the olfactory bulb and in olfaction. Physiol Behav 77:607--612

16. Uchida N, Takahashi YK, Tanifuji M, Mori K (2000) Odor maps in the mammalian olfactory bulb: domain organization and odorant structural features. Nat Neurosci 3:1035-1043

17. Dhawale AK, Hagiwara A, Bhalla US, Murthy VN, Albeanu DF (2010) Non-redundant odor coding by sister mitral cells revealed by light addressable glomeruli in the mouse. Nat Neurosci 13:14041412

18. Mombaerts P, Wang F, Dulac C et al. (1996) Visualizing an olfactory sensory map. Cell 87:675-686

19. Tan J, Savigner A, Ma M, Luo M (2010) Odor information processing by the olfactory bulb analyzed in gene-targeted mice. Neuron 65:912-926

20. Adrian ED (1950) The electrical activity of the mammalian olfactory bulb. Electroencephalogr Clin Neurophysiol 2:377-388

21. Cang J, Isaacson JS (2003) In vivo whole-cell recording of odor-evoked synaptic transmission in the rat olfactory bulb. J Neurosci 23:4108-4116

22. Macrides F, Chorover SL (1972) Olfactory bulb units: activity correlated with inhalation cycles and odor quality. Science 175:84-87

23. Margrie TW, Schaefer AT (2003) Theta oscillation coupled spike latencies yield computational vigour in a mammalian sensory system. J Physiol 546(Pt 2):363-374

24. Shepherd GM, Greer CA (1990) Olfactory bulb. Synaptic organization of the brain. Oxford University Press, New York, pp 133-169

25. Sauer B, Henderson N (1988) Site-specific DNA recombination in mammalian cells by the Cre recombinase of bacteriophage P1. Proc Natl Acad Sci U S A 85:5166-5170 
A1

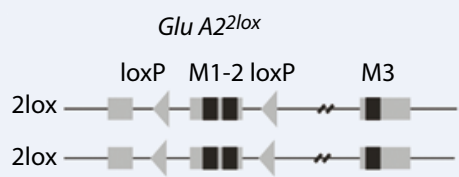

Virus injection

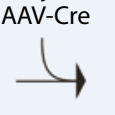

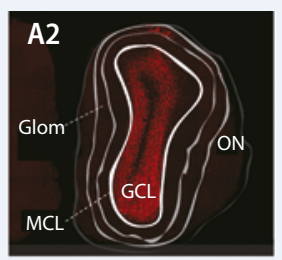

B1

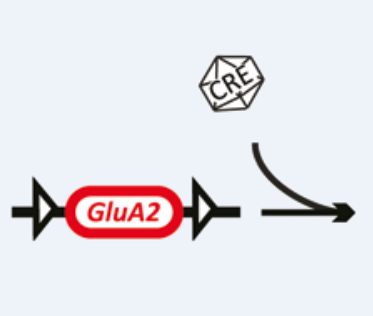

C1

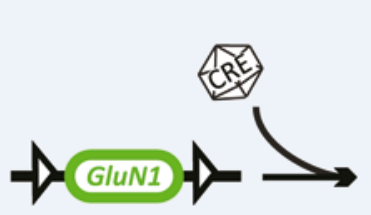

B2 GluA2 $\triangle G C L$

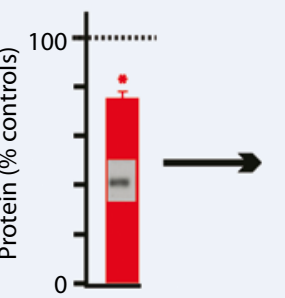

C2

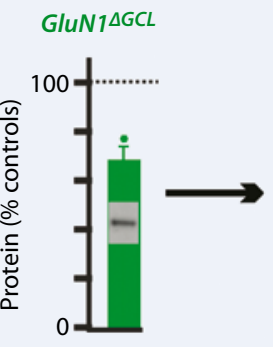

B3

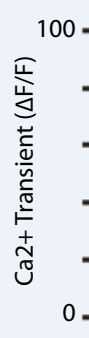

B4

C3
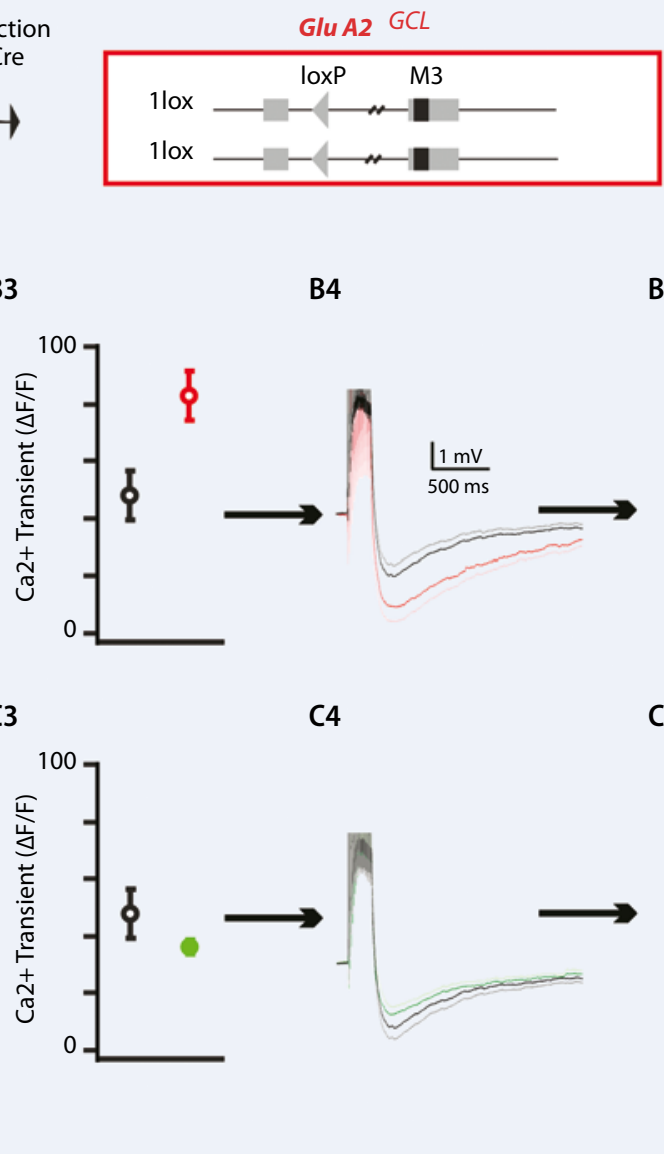

B5

C5

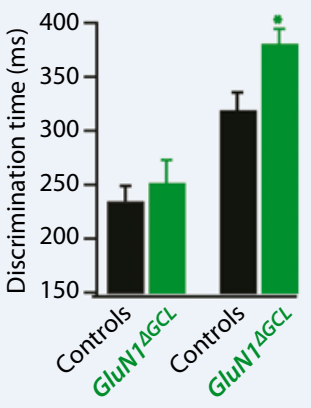

Fig. $6 \Delta$ A Strategy for the targeted genetic modification of granule cells. A1 Starting point: a transgenic mouse in which the target gene (here the AMPA receptor subunit GluA2) is flanked by the "loxP" interface in a targeted manner. By means of Crerecombinase expression, DNA on loxP sites is dissected, resulting in the elimination of the target gene in the cells expressing Cre-recombinase. Cre expression takes place following the targeted injection of an adeno-associated virus ( $A A V$ - $C r e$ ) functioning as a gene shuttle. A2 By means of targeted injection at several sites in the olfactory bulb, infection and thus Cre expression can be limited to cells in the granule layer (GCL). $M C L$ mitral cell layer, Glom glomerular layer, $O N$ olfactory nerve. Red virus-mediated Cre expression. B Virus-mediated elimination of GluA2 in granule cells (B1) causes a reduction in GluA2 protein (B2), increased calcium influx in granule cell synapses (measured using two-photon microscopy in brain sections, B3), reduced inhibition (whole-cell recording of mitral cells enables the measurement of recurrent inhibition, B4; see also - Fig. 2B) and, ultimately, enhanced olfactory discrimination of similar mixtures (shorter discrimination time in the behaviour experiment, B5). C The analogous result for the elimination of the NMDA receptor subunit GluN1, resulting in reduced calcium influx, reduced inhibition and worsened olfactory discrimination (modified from [27])

26. Tsien JZ, Chen DF, Gerber D et al. (1996) Subregion- and cell type-restricted gene knockout in mouse brain. Cell 87:1317-1326

27. Abraham NM, Egger V, Shimshek DR et al. (2010) Synaptic inhibition in the olfactory bulb accelerates odor discrimination in mice. Neuron 65:399411

28. Isaacson JS (2001) Mechanisms governing dendritic gamma-aminobutyric acid (GABA) release in the rat olfactory bulb. Proc Natl Acad Sci U S A 98:337342

29. Nusser Z, Kay LM, Laurent G, Homanics GE, Mody I (2001) Disruption of GABA(A) receptors on GABAergic interneurons leads to increased oscillatory power in the olfactory bulb network. J Neurophysiol 86:2823-2833

30. Shimshek DR, Bus T, Kim J et al. (2005) Enhanced odor discrimination and impaired olfactory memory by spatially controlled switch of AMPA receptors. PLoS Biol 3:e354
31. Bodyak N, Slotnick B (1999) Performance of mice in an automated olfactometer: odor detection, discrimination and odor memory. Chem Senses 24:637-645 